\title{
The Operator Algebra of the Quantum Relativistic Oscillator
}

\author{
Ion I. Cotăescu \\ Gheorghe Drăgănescu \\ The West University of Timişoara, \\ V. Pârvan Ave. 4, RO-1900 Timişoara, Romania
}

February 1, 2018

\begin{abstract}
The operator algebras of a new family of relativistic geometric models of the relativistic oscillator [1] are studied. It is shown that, generally, the operator of number of quanta and the pair of the shift operators of each model are the generators of a non-unitary representation of the $s o(1,2)$ algebra, except a special case when this algebra becomes the standard one of the non-relativistic harmonic oscillator.

Pacs: 04.62.+v, 03.65.Ge
\end{abstract}

\section{Introduction}

In the general relativity, the geometric models play the role of kinematics, helping us to understand the characteristics of the classical or quantum free motion on a given background. One of the simplest $(1+1)$ geometric models is that of the (classical or quantum) relativistic harmonic oscillator ( $\mathrm{RHO}$ ). Based on phenomenological [2] and group theoretical [3, [1] arguments, this has been defined as a free system on the anti-de Sitter static background. There exists a $(3+1)$ anti-de Sitter static metric [2] which can be restricted 
to the $(1+1)$ metric given by the line element

$$
d s^{2}=\frac{1}{1-\omega^{2} x^{2}} d t^{2}-\frac{1}{\left(1-\omega^{2} x^{2}\right)^{2}} d x^{2} .
$$

This metric reproduces the classical equation of motion of the non-relativistic harmonic oscillator (NRHO) of the frequency $\omega$. Moreover, the corresponding quantum model, represented by a free scalar massive quantum field, has an equidistant discrete spectrum with a ground state energy larger, but approaching $\omega / 2$ in the non-relativistic limit (in natural units $\hbar=c=1$ ) [5]. In a previous article [1] we have generalized this model to the family of models depending on a real parameter $\lambda$ which has the metrics given by

$$
d s^{2}=g_{00} d t^{2}+g_{11} d x^{2}=\frac{1+(1+\lambda) \omega^{2} x^{2}}{1+\lambda \omega^{2} x^{2}} d t^{2}-\frac{1+(1+\lambda) \omega^{2} x^{2}}{\left(1+\lambda \omega^{2} x^{2}\right)^{2}} d x^{2} .
$$

Here, the parametrization has been defined in order to obtain the exact antide Sitter metric (1) for $\lambda=-1$. We have shown [1] that the quantum models with $\lambda>0$ have mixed energy spectra, with a finite discrete sequence and a continuous part, while for $\lambda \leq 0$ these spectra are countable. However, despite of their different relativistic behavior, all these models have the same non-relativistic limit, namely the NRHO. For this reason we shall use the name of relativistic oscillators ( $\mathrm{RO})$ for all the models with $\lambda \neq-1$, understanding that the RHO is only that of the anti-de Sitter metric.

In this article, we intend to study the operator algebra of the quantum RO with countable energy spectra. For these models $\lambda \leq 0$ and, therefore, it is convenient to denote

$$
\lambda=-\epsilon^{2}, \quad \hat{\omega}=\epsilon \omega,
$$

and to rewrite our previous results in this new notation. We take $\epsilon \geq 0$ so that the pure anti-de Sitter RHO will have $\epsilon=1$.

The energy spectra of our quantum models depend on only one quantum number [1]. Therefore, we will have one operator of number of quanta and a pair of shift (i.e. raising or lowering) operators. Our main objective is to identify the algebra which is linearly generated by these operators. To this end, first we show that there exists a natural (holonomic) frame where the relativistic energy eigenfunctions coincide with those of the non-relativistic Pöschl-Teller (PT) system [6, 7]. By using their known properties, we shall 
construct the main operators which act on the one-particle wave functions, giving a special attention to the three mentioned ones. The result is that for all the RO with $\epsilon>0$, including the RHO, these are the generators of the $s o(1,2)$ algebra which has as Casimir operator just the Klein-Gordon equation. In the limit $\epsilon \rightarrow 0$ the $s o(1,2)$ algebra degenerates in the standard algebra of the NRHO.

We start, in the second section, with a short review of the properties of the Hilbert space of one-particle states of the free quantum scalar massive field in the coordinate representation. In the next section we briefly present our previous results concerning the RO, giving the energy levels and the energy eigenfunctions with their normalization factors. In the Sec. 4 we show that, in the special frame where the metric is the conformal transformation of the Minkowski flat metric, the RO generates a relativistic PT problem for which the shift operators of the energy basis are known [7]. Based on these results we analyze, in the next section, the operator algebra of the models with $\epsilon>0$, obtaining a non-unitary representation of the $s o(1,2)$ algebra which is equivalent to an unitary one. The case of $\epsilon=0$ is studied in the Sec. 6 .

\section{Preliminaries}

Let us consider a static background with the natural frame $(t, x)$ in which the metric is $g_{\mu \nu}(x)$, with $\mu, \nu=0,1$. We shall assume that this is symmetric with respect to $x=0$ and we shall denote $g=\operatorname{det}\left(g_{\mu \nu}\right)$. The domain, $D$, of the free motion observed by an observer situated at $x=0$ is that bounded by the observer's event horizon. In general, this is $D=\left(-x_{e}, x_{e}\right)$ where $\pm x_{e}$ are either the finite points where the metric is singular or $\pm \infty$ in the case of the regular metrics. On this domain, we shall define the scalar field $\phi$ of the mass $m$, supposing that this is minimally coupled with the gravitational field [8]. In the case of static backgrounds the energy is conserved. Therefore, the Klein-Gordon equation

$$
\frac{1}{\sqrt{-g}} \partial_{\mu}\left(\sqrt{-g} g^{\mu \nu} \partial_{\nu} \phi\right)+m^{2} \phi=0,
$$

admits a set of fundamental solutions (of positive and negative frequency) of 
the form

$$
\phi_{E}^{(+)}=\frac{1}{\sqrt{2 E}} e^{-i E t} U_{E}(x), \quad \phi^{(-)}=\left(\phi^{(+)}\right)^{*} .
$$

These must be orthogonal with respect to the relativistic scalar product [8]

$$
\left(\phi, \phi^{\prime}\right)=i \int_{D} d x \sqrt{-g} g^{00} \phi^{*} \stackrel{\leftrightarrow}{\partial_{0}} \phi^{\prime}
$$

which, in fact, reduces to the following scalar product of the wave functions $U$

$$
\left(U, U^{\prime}\right)=\int_{D} d x \mu(x) U^{*}(x) U^{\prime}(x)
$$

where

$$
\mu=\sqrt{-g} g^{00} .
$$

Now we observe that, according to (5), the one-particle state space, $H$, coincides to that of the antiparticle. In the coordinate representation this is the space $L^{2}(D, \mu)$ of the square integrable functions with respect to the scalar product (7). All these functions must satisfy the condition

$$
\lim _{x \rightarrow \pm x_{e}} U(x)=0
$$

which is obvious for $x_{e}=\infty$. Moreover, when $x_{e}$ is finite then the metric as well as the weight function $\mu$ are singular at $\pm x_{e}$ and, consequently, the condition (9) is also necessary. A set of wave functions, $U_{n}, n=0,1, \ldots$, represents a countable basis in $H$ if these are orthonormal,

$$
\left(U_{n}, U_{n^{\prime}}\right)=\delta_{n, n^{\prime}}
$$

and satisfy the completeness relation

$$
\sum_{n} U_{n}{ }^{*}(x) U_{n}\left(x^{\prime}\right)=\frac{1}{\mu(x)} \delta\left(x-x^{\prime}\right) .
$$

The linear operators on $H$ will be denoted using boldface. They can be defined either by giving their matrix elements in a countable basis or as differential operators in the coordinate representation. The most general differential operator we shall use here will have the form

$$
(\mathbf{D} U)(x)=i\left[f(x) \frac{d}{d x}+h(x)\right] U(x),
$$


depending on two arbitrary real functions $f$ and $h$. Its adjoint with respect to the scalar product (团) is

$$
\mathbf{D}^{+}=\mathbf{D}+i\left[\frac{1}{\mu} \frac{d(\mu f)}{d x}-2 h\right] \mathbf{1}
$$

where 1 is the unit operator. Hereby, we see that for $h=\partial_{x}(\mu f) / 2 \mu$ the operator $\mathbf{D}$ is selfadjoint.

In general, any $(1+1)$ static background admits a special natural frame, $(t, \hat{x})$, in which the metric is a conformal transformation of the Minkowski flat metric. This new frame can be obtained by changing the space coordinate

$$
x \rightarrow \hat{x}=\int d x \mu(x)+\text { const },
$$

so that

$$
\hat{g}_{00}(\hat{x})=-\hat{g}_{11}(\hat{x})=\sqrt{-\hat{g}(\hat{x})}
$$

and $\hat{\mu}(\hat{x})=1$. Then, from (13) it results that the momentum operator $\sim i \partial_{\hat{x}}$ is selfadjoint. The state space $H$ is represented now by $L^{2}(\hat{D})$ where $\hat{D}$ is the domain of the new space coordinate corresponding to $D$. It is obvious that both the spaces $L^{2}(D, \mu)$ and $L^{2}(\hat{D})$ come from the same coordinate representation of the space $H$ since the change of the continuous parameter of a generalized basis changes only the normalization scale.

\section{Relativistic oscillators}

Let us first discuss the general case of $\epsilon>0$ and then turn to the limit $\epsilon \rightarrow 0$. In the frames $(t, x)$, the metrics are given by (2) where we have to change the parameters according to (3). For the models with $\epsilon>0$ these metrics are singular at $\pm 1 / \hat{\omega}$ so that $D=(-1 / \hat{\omega}, 1 / \hat{\omega})$.

The Klein-Gordon equation can be put in the form

$$
\left(-\left(1-\hat{\omega}^{2} x^{2}\right) \frac{d^{2}}{d x^{2}}+\hat{\omega}^{2} x \frac{d}{d x}+\frac{m^{2}}{\epsilon^{2}} \frac{\hat{\omega}^{2} x^{2}}{1-\hat{\omega}^{2} x^{2}}\right) U(x)=\left(E^{2}-m^{2}\right) U(x)
$$

while the weight function which defines the scalar product (7) is

$$
\mu(x)=\frac{1}{\sqrt{1-\hat{\omega}^{2} x^{2}}} .
$$


Since the energy spectrum is countable, the energy eigenfunctions are the square integrable solutions of (16). These can be written in terms of hypergeometric functions as [1]

$$
U_{n}(x)=N_{n_{s}, s}\left(1-\hat{\omega}^{2} x^{2}\right)^{\frac{k}{2}} x^{s} F\left(-n_{s}, k+s+n_{s}, s+\frac{1}{2}, \hat{\omega}^{2} x^{2}\right),
$$

where the parameter

$$
k=\frac{1}{2}\left[1+\sqrt{1+4 \frac{m^{2}}{\epsilon^{2} \hat{\omega}^{2}}}\right]>1
$$

is the positive solution of the equation

$$
k(k-1)=\frac{m^{2}}{\epsilon^{2} \hat{\omega}^{2}} .
$$

The quantum numbers, $n_{s}=0,1,2 \ldots$ and $s=0,1$, can be embedded into the main quantum number $n=2 n_{s}+s$. This will take even values if $s=0$ and odd values for $s=1$. Hence, the functions $U_{n}(x)$ are real polynomials of the degree $n$ in $x$, with the factor $\left(1-\hat{\omega}^{2} x^{2}\right)^{k / 2}$, which assures the condition (9). The normalization factors can be easily calculated in terms of Jacobi polynomials [9]. The result is

$$
N_{n_{s}, s}=(-1)^{n_{s}} \frac{(\hat{\omega})^{s+\frac{1}{2}}}{\sqrt{n_{s} !}}\left(s+\frac{1}{2}\right)_{n_{s}}\left[\frac{\left(k+s+2 n_{s}\right) \Gamma\left(k+s+n_{s}\right)}{\Gamma\left(n_{s}+s+\frac{1}{2}\right) \Gamma\left(n_{s}+k+\frac{1}{2}\right)}\right]^{\frac{1}{2}}
$$

where we have used the notation $(z)_{n}=z(z+1) \ldots(z+n-1)$. Moreover, the normalized energy eigenfuncions can also be written in terms of associated Legendre polynomials [7].

The energy levels result from the quantization condition [1],

$$
E_{n}^{2}-m^{2}\left(1-\frac{1}{\epsilon^{2}}\right)=\hat{\omega}^{2}(k+n)^{2}
$$

which gives

$$
E_{n}^{2}=m^{2}+\hat{\omega}^{2}\left[2 k\left(n+\frac{1}{2}\right)+n^{2}\right], \quad n=0,1,2 \ldots
$$


for $\epsilon \neq 1$, and

$$
E_{n}=\hat{\omega}(k+n)
$$

in the case of the RHO [5], when $\epsilon=1$.

We can conclude that our RO with $\epsilon>0$ are systems of massive scalar particles confined to wells. Their properties are determined by three parameters, $m, \omega$ and $\epsilon$. This last one is our new parameter which gives the desired well width, $2 / \epsilon \omega$, when the frequecy $\omega$ is fixed. It is interesting that all these parameters are concentrated in the expression of $k$ so that the eigenfunctions (18) depend only on $\hat{\omega}=\epsilon \omega$ and $k$ while the energies (23) involve all of them. Thus there is a possibility to have RO with different energy spectra but having the same energy eigenfunctions.

\section{The relativistic Pöschl-Teller problem}

Now we shall change the space coordinate according to (14) where $\mu$ is given by (17). We obtain

$$
\hat{x}=\frac{1}{\hat{\omega}} \arcsin \hat{\omega} x .
$$

In the new frame $(t, \hat{x})$ the line element is

$$
d s^{2}=\left(1+\frac{1}{\epsilon^{2}} \tan ^{2} \hat{\omega} \hat{x}\right)\left(d t^{2}-d \hat{x}^{2}\right)
$$

and $\hat{D}=(-\pi / 2 \hat{\omega}, \pi / 2 \hat{\omega})$. The Klein-Gordon equation takes the form

$$
\left(-\frac{d^{2}}{d \hat{x}^{2}}+\frac{m^{2}}{\epsilon^{2}} \tan ^{2} \hat{\omega} \hat{x}\right) U_{n}(\hat{x})=\left(E_{n}{ }^{2}-m^{2}\right) U_{n}(\hat{x}) .
$$

The second term of its left-hand side can be rewritten using (20):

$$
V_{P T}(\hat{x})=k(k-1) \hat{\omega}^{2} \tan ^{2} \hat{\omega} \hat{x} .
$$

This will be called the relativistic (symmetric) PT potential since the solutions $(18)$ in the new variable $\hat{x}$,

$$
U_{n}(\hat{x})=N_{n_{s}, s} \hat{\omega}^{-s} \cos ^{k} \hat{\omega} \hat{x} \sin ^{s} \hat{\omega} \hat{x} F\left(-n_{s}, k+s+n_{s}, s+\frac{1}{2}, \sin ^{2} \hat{\omega} \hat{x}\right),
$$


coincide with those given by the non-relativistic PT potential $V_{P T} / 2 m$. Of course, the energies, as well as the significance of the parameters, differ from those of the non-relativistic case.

On the other hand, we know that the functions (29) represent a complete set of orthonormal functions in $L^{2}(\hat{D})$. This means that the set $U_{n}, n=0,1$, . is a countable basis in $H$, namely the energy basis. Its shift operators [7]

$$
\begin{aligned}
& \left(\mathbf{A}_{(+)} U_{n}\right)(\hat{x})=\frac{1}{\hat{\omega} \sqrt{2 k}}\left[-\cos \hat{\omega} \hat{x} \frac{d}{d \hat{x}}+\hat{\omega} \sin \omega \hat{x}(k+n)\right] U_{n}(\hat{x}), \\
& \left(\mathbf{A}_{(-)} U_{n}\right)(\hat{x})=\frac{1}{\hat{\omega} \sqrt{2 k}}\left[\cos \hat{\omega} \hat{x} \frac{d}{d \hat{x}}+\hat{\omega} \sin \omega \hat{x}(k+n)\right] U_{n}(\hat{x})
\end{aligned}
$$

have the action

$$
\mathbf{A}_{(+)} U_{n}=C_{n}^{(+)} U_{n+1}, \quad \mathbf{A}_{(-)} U_{n}=C_{n}^{(-)} U_{n-1},
$$

where

$$
\begin{aligned}
C_{n}^{(+)} & =\frac{1}{\sqrt{2 k}}\left(\frac{(2 k+n)(k+n)}{k+n+1}\right)^{\frac{1}{2}} \sqrt{n+1}, \\
C_{n}^{(-)} & =\frac{1}{\sqrt{2 k}}\left(\frac{(2 k+n-1)(k+n)}{k+n-1}\right)^{\frac{1}{2}} \sqrt{n} .
\end{aligned}
$$

We note that according to (13) the shift operators are not related between them through Hermitian conjugation, i.e. $\mathbf{A}_{( \pm)}^{+} \neq \mathbf{A}_{(\mp)}$.

\section{Algebra}

\subsection{The differential operators}

Let us consider the position and momentum selfadjoint operators,

$$
(\hat{\mathbf{X}} U)(\hat{x})=\hat{x} U(\hat{x}), \quad(\hat{\mathbf{P}} U)(\hat{x})=i \frac{d U(\hat{x})}{d \hat{x}},
$$

in the frame $(t, \hat{x})$, with the commutation rule

$$
[\hat{\mathbf{P}}, \hat{\mathbf{X}}]=i \mathbf{1} \text {. }
$$


Then, from (27) we see that the energy square operator is

$$
\mathbf{E}^{2}=m^{2} \mathbf{1}+\hat{\mathbf{P}}^{2}+\frac{m^{2}}{\epsilon^{2}} \tan ^{2} \hat{\omega} \hat{\mathbf{X}}
$$

Its form suggests us to introduce the pair of adjoint operators [10]

$$
\begin{gathered}
\tilde{\mathbf{a}}=\frac{1}{\hat{\omega} \sqrt{2 k}}(-i \hat{\mathbf{P}}+k \hat{\omega} \tan \hat{\omega} \hat{\mathbf{X}}), \\
\tilde{\mathbf{a}}^{+}=\frac{1}{\hat{\omega} \sqrt{2 k}}(i \hat{\mathbf{P}}+k \hat{\omega} \tan \hat{\omega} \hat{\mathbf{X}}) .
\end{gathered}
$$

which satisfy the commutation relation

$$
\left[\tilde{\mathbf{a}}, \tilde{\mathbf{a}}^{+}\right]=\mathbf{1}+\frac{1}{2 k}\left(\tilde{\mathbf{a}}^{+}+\tilde{\mathbf{a}}\right)^{2}
$$

and allow to write

$$
\mathbf{E}^{2}=m^{2} \mathbf{1}+2 k \hat{\omega}^{2}\left(\tilde{\mathbf{a}}^{+} \tilde{\mathbf{a}}+\frac{1}{2} \mathbf{1}\right) .
$$

The commutation relations of $\mathbf{E}^{2}$ with $\hat{\mathbf{X}}$ and $\hat{\mathbf{P}}$ can also be calculated from (36) and (37). Another exercise is to replace the position operator by the effective position operator

$$
\hat{\mathbf{X}}_{e f}=\frac{1}{\hat{\omega}} \tan \hat{\omega} \hat{\mathbf{X}}
$$

in order to recover the familiar formulas

$$
\hat{\mathbf{X}}_{e f}=\frac{1}{\hat{\omega} \sqrt{2 k}}\left(\tilde{\mathbf{a}}^{+}+\tilde{\mathbf{a}}\right), \quad \hat{\mathbf{P}}=-i \hat{\omega} \sqrt{\frac{k}{2}}\left(\tilde{\mathbf{a}}^{+}-\tilde{\mathbf{a}}\right) .
$$

\subsection{The non-differential operators}

However, there are other operators which are not differential. The analysis of their structure can be done by using the operators of the standard oscillator algebra, $\mathbf{a}, \mathbf{a}^{+}$(with $\left[\mathbf{a}, \mathbf{a}^{+}\right]=\mathbf{1}$ ) and $\mathbf{N}=\mathbf{a}^{+} \mathbf{a}$, which can be defined in the energy basis as follows:

$$
\mathbf{a}^{+} U_{n}=\sqrt{n+1} U_{n+1}, \quad \mathbf{a} U_{n}=\sqrt{n} U_{n-1}, \quad \mathbf{N} U_{n}=n U_{n} .
$$


Now the quantization condition (22) becomes

$$
\mathbf{E}^{2}=\hat{\omega}^{2}\left[(\mathbf{N}+k \mathbf{1})^{2}+\left(\epsilon^{2}-1\right) k(k-1) \mathbf{1}\right]
$$

while the shift operators can be put in the form

$$
\begin{aligned}
& \mathbf{A}_{(+)}=(\cos \hat{\omega} \hat{\mathbf{X}}) \tilde{\mathbf{a}}^{+}+\frac{1}{\sqrt{2 k}}(\sin \hat{\omega} \hat{\mathbf{X}}) \mathbf{N} \\
& \mathbf{A}_{(-)}=(\cos \hat{\omega} \hat{\mathbf{X}}) \tilde{\mathbf{a}}+\frac{1}{\sqrt{2 k}}(\sin \hat{\omega} \hat{\mathbf{X}}) \mathbf{N}
\end{aligned}
$$

Furthermore, from Eqs. (30) - (34) it results that these operators can be expressed in terms of the generators of the standard oscillator algebra as

$$
\mathbf{A}_{(+)}=\mathbf{a}^{+} w_{(+)}(\mathbf{N}), \quad \mathbf{A}_{(-)}=w_{(-)}(\mathbf{N}) \mathbf{a},
$$

where

$$
w_{(+)}(\mathbf{N})=w_{(-)}(\mathbf{N}) \frac{\mathbf{N}+k \mathbf{1}}{\mathbf{N}+k \mathbf{1}+\mathbf{1}}=\left[\frac{(\mathbf{N}+2 k \mathbf{1})(\mathbf{N}+k \mathbf{1})}{2 k(\mathbf{N}+k \mathbf{1}+\mathbf{1})}\right]^{\frac{1}{2}} .
$$

Hereby, we obtain the commutation relations

$$
\left[\mathbf{A}_{(-)}, \mathbf{A}_{(+)}\right]=\mathbf{1}+\frac{1}{k} \mathbf{N}, \quad\left[\mathbf{N}, \mathbf{A}_{( \pm)}\right]= \pm \mathbf{A}_{( \pm)}
$$

and the identity

$$
2 k \mathbf{A}_{(+)} \mathbf{A}_{(-)}=\mathbf{N}[\mathbf{N}+(2 k-1) \mathbf{1}]
$$

which is nothing else than the operator form of the Klein-Gordon equation, as it results fom (30), (31) and (44).

Let us observe that the operators $\sqrt{2 k} \mathbf{A}_{(+)}, \sqrt{2 k} \mathbf{A}_{(-)}$and $\mathbf{N}+k \mathbf{1}$ are the generators of a non-unitary representation of the $s o(1,2)$ algebra. This is (non-unitary) equivalent with the unitary representation of the lowest weight $k$, which has the generators

$$
\begin{aligned}
\mathbf{K}_{(+)} & =\mathbf{K}_{1}+i \mathbf{K}_{2}= \\
& =\sqrt{2 k}(\mathbf{N}+k \mathbf{1})^{\frac{1}{2}} \mathbf{A}_{(+)}(\mathbf{N}+k \mathbf{1})^{-\frac{1}{2}}=\mathbf{a}^{+}(\mathbf{N}+2 k \mathbf{1})^{\frac{1}{2}} \\
\mathbf{K}_{(-)} & =\mathbf{K}_{1}-i \mathbf{K}_{2}= \\
& =\sqrt{2 k}(\mathbf{N}+k \mathbf{1})^{\frac{1}{2}} \mathbf{A}_{(-)}(\mathbf{N}+k \mathbf{1})^{-\frac{1}{2}}=(\mathbf{N}+2 k \mathbf{1})^{\frac{1}{2}} \mathbf{a} \\
\mathbf{K}_{3} & =\mathbf{N}+k \mathbf{1} .
\end{aligned}
$$


The Casimir operator

$$
\mathbf{K}_{3}{ }^{2}-\mathbf{K}_{1}{ }^{2}-\mathbf{K}_{2}{ }^{2}=k(k-1) \mathbf{1}
$$

is an alternative form of the Klein-Gordon operator (51).

Finally we must specify that the operators $\hat{\mathbf{X}}$ and $\hat{\mathbf{P}}$ can be expressed in terms of $\mathbf{a}$ and $\mathbf{a}^{+}$by using the Eqs. (30), (31) and (48). However, we can introduce other coordinate and momentum operators corresponding to all the natural frames we desire to choose. Obviously, all these operators are analytic functions of $\hat{\mathbf{X}}\left(\mathbf{a}, \mathbf{a}^{+}\right)$and $\hat{\mathbf{P}}\left(\mathbf{a}, \mathbf{a}^{+}\right)$. Thus it results that the whole operator algebra of the RO is freely generated by $\mathbf{a}$ and $\mathbf{a}^{+}$only.

\section{The limit $\epsilon \rightarrow 0$}

The case of $\epsilon=0$ can be solved separately [1]. Here we have $x=\hat{x}$ and $x_{e} \rightarrow \infty$ so that, in the coordinate representation, $H$ will appear as $L^{2}(R)$. The solutions of the Klein-Gordon equation,

$$
-\frac{d^{2} U_{n}^{0}}{d x^{2}}+m^{2} \omega^{2} x^{2} U_{n}^{0}=\left(E_{n}{ }^{2}-m^{2}\right) U_{n}^{0}
$$

coincide with the familiar energy eigenfunctions of the NRHO, while the energy spectrum is given by

$$
E_{n}{ }^{2}=m^{2}+2 m \omega\left(n+\frac{1}{2}\right), \quad n=0,1,2, \ldots
$$

On the other hand, we have shown [1] that the solutions of our RO are continuous in $\epsilon=0$ in the sense that the limit of the energy eigenfunction (18) for $\epsilon \rightarrow 0$, calculated up to the normalization factors, gives just the solutions of (56). Now we can convince ourselves, that the normalization factors (21) also behave correct in the limit $\epsilon \rightarrow 0$, giving the usual normalization factors of the NRHO energy eigenfunctions, $U_{n}^{0}$, which should satisfy $\left(U_{n}^{0}, U_{n^{\prime}}^{0}\right)=$ $\delta_{n n^{\prime}}$. Indeed, by taking into account that in this limit we have $\hat{\omega} \rightarrow 0$, $k \rightarrow \infty$ but $\epsilon^{2} k \rightarrow m / \omega$, and by using the asymptotic form of the functions $\Gamma(z)$ for large $z[9]$, we find that

$$
\lim _{\epsilon \rightarrow 0} U_{n}(x)=\left(\frac{m \omega}{\pi}\right)^{\frac{1}{4}} \frac{(-1)^{n_{s}}(s+1)}{2^{n_{s}+\frac{s}{2}} n_{s} !} \sqrt{\left(2 n_{s}+s\right) !} \times
$$




$$
\begin{array}{r}
e^{-m \omega x^{2} / 2}(\sqrt{m \omega} x)^{s} F\left(-n_{s}, s+\frac{1}{2}, m \omega x^{2}\right)= \\
=\left(\frac{m \omega}{\pi}\right)^{\frac{1}{4}} \frac{1}{\sqrt{n ! 2^{n}}} e^{-m \omega x^{2} / 2} H_{n}(\sqrt{m \omega} x)=U_{n}^{0}(x),
\end{array}
$$

where $H_{n}$ are the Hermite polynomials and $n=2 n_{s}+s$ as defined above. We note that this result justifies the choice of the phase factor $(-1)^{n_{s}}$ of (21).

The behavior of the energy eigenfunctions suggests us to derive the operator algebra in the case of $\epsilon=0$ as the limit of the algebra obtained in the previous section. We observe that $w_{( \pm)}(\mathbf{N}) \rightarrow \mathbf{1}$ when $\epsilon \rightarrow 0$ so that

$$
\lim _{\epsilon \rightarrow 0} \mathbf{A}_{(+)}=\lim _{\epsilon \rightarrow 0} \tilde{\mathbf{a}}^{+}=\mathbf{a}^{+}, \quad \lim _{\epsilon \rightarrow 0} \mathbf{A}_{(-)}=\lim _{\epsilon \rightarrow 0} \tilde{\mathbf{a}}=\mathbf{a} .
$$

Furthermore, from (30) and (31) we see that the operators a and $\mathbf{a}^{+}$become differential operators of the form

$$
\begin{array}{r}
(\mathbf{a} U)(x)=\frac{1}{\sqrt{2 m \omega}}\left(\frac{d}{d x}+m \omega x\right) U(x), \\
\left(\mathbf{a}^{+} U\right)(x)=\frac{1}{\sqrt{2 m \omega}}\left(-\frac{d}{d x}+m \omega x\right) U(x) .
\end{array}
$$

This means that the position and the momentum operators can be written as in the non-relativistic case,

$$
\mathbf{X}=\frac{1}{\sqrt{2 m \omega}}\left(\mathbf{a}^{+}+\mathbf{a}\right), \quad \mathbf{P}=-i \sqrt{\frac{m \omega}{2}}\left(\mathbf{a}^{+}-\mathbf{a}\right) .
$$

Hence, the conclusion is that the $s o(1,2)$ algebra of the models with $\epsilon>0$ degenerates in the usual NRHO algebra when $\epsilon \rightarrow 0$. Simultaneously, the Klein-Gordon operator (51) becomes $\mathbf{a}^{+} \mathbf{a}=\mathbf{N}$. Thus, for $\epsilon=0$ the energy eigenfunctions as well as the shift operators are the same as those of the NRHO. The unique difference is the formula of the energy levels which gives the relativistic energy square operator

$$
\mathbf{E}^{2}=m^{2} \mathbf{1}+2 m \omega\left(\mathbf{N}+\frac{1}{2} \mathbf{1}\right)
$$

and new commutation rules for $\mathbf{E}^{2}$ with $\mathbf{X}$ or $\mathbf{P}$. 


\section{Comments}

In this article we have studied the properties of the fundamental solutions of the Klein-Gordon equations of the RO by using the traditional methods of the coordinate representation. This allowed us to study the form of the energy eigenfunctions in two natural frames. The first one, $(t, x)$, is important since here the classical equations of motion of the RO look like that of the NRHO. This indicates that the physical meaning of the relativistic behavior of the RO could be better point out in this frame. The other frame, $(t, \hat{x})$, offers the advantage of the simplest form of the Klein-Gordon equation (27). We have shown that for $\epsilon>0$ this is the relativistic version of the PT system. Moreover, we have seen that in the limit $\epsilon \rightarrow 0$ both these frames coincide while the solutions of the Klein-Gordon equation become just those of the NRHO. Thus we can conclude that, at least in the frame $(t, \hat{x})$, the space behavior of the RO remains very closed to that of several non-relativistic systems. However, the operators of physical interest have new properties and specific and consistent, although quit complicated, commutation relations. We must specify that our results concerning the commutation rules of the energy, position and momentum operators differ from those predicted in the algebraic approach of the RHO [3]. What is remarkable here is that we have recovered on an other way the $s o(1,2)$ algebra, for all the RO with $\epsilon>0$, so that the Klein-Gordon operator should be just its Casimir operator. As mentioned, all these systems are of massive scalar particles confined to wells having the width $2 / \epsilon \omega$ in the frame $(t, x)$ or $\pi / \epsilon \omega$ in the frame $(t, \hat{x})$.

Finally we note that all the results we have obtained could be the starting point of the construction of the quantum field theory of the scalar field $\phi$. This must be defined on $H$ with values in the field operator algebra by introducing suitable creation and annihilation operators. Moreover, all the operators we have discussed here will generate the one-particle operators of the quantum field theory. Then the physical meaning of these operators will be better understood because of the possibility of analyzing their time evolution with the help of the Hamiltonian operator. In our opinion, in this way one could obtain new answers in some sensitive problems such as that

related to the definition of the relativistic position and momentum operators. 


\section{References}

[1] I. I. Cotaescu, Geometric Models of the Relativistic Harmonic Oscillators, to appear in Int. J. Mod. Phys. A.

[2] E. van Beveren, G. Rupp, T. A. Rijken, C. Dullemond, Phys. Rev. D 27, 1527 (1983); C. Dullemond, E. van Beveren, Phys. Rev. D 28, 1028 (1983)

[3] V. Aldaya, J. Bisquert, J. Navarro-Salas, Phys. Lett. A156, 315 (1991)

[4] V. Aldaya. J. A. de Azcarraga, J. Math. Phys. 23, 1297 (1982); V. Aldaya, J. Bisquert, R. Loll, J. Navarro-Salas, J. Math. Phys. 33, 3087 (1992)

[5] D. J. Navarro, J. Navarro-Salas, preprint HEP-TH-9406001

[6] G. Pöschl, E. Teller, Z. Phys. 83, 149 (1933)

[7] M. M. Nieto, L. M. Simmons Jr., Phys. Rev. Lett. 41, 207 (1978); Phys. Rev. D 20, 1332 (1979)

[8] N. D, Birrel, P. C. W. Davies, Quantum Fields in Curved Space, Cambridge University Press, Cambridge (1982)

[9] M. Abramowitz, I. A. Stegun, Handbook of Mathematical Functions, Dover, New York (1964)

[10] R. Dutt, A. Khare, U. P. Sukhatme, Am. J. Phys. 56, 163 (1987) 